\title{
MODULUS OF CONVEXITY, CHARACTERISTIC OF CONVEXITY AND FIXED POINT THEOREMS
}

\author{
BY HAJIME ISHIHARA AND WATARU TAKAHASHI
}

\section{§1. Introduction.}

Let $C$ be a bounded closed convex subset of a Banach space $E$ and let $T$ be a nonexpansive mapping from $C$ into itself. Browder [2] and Göhde [10] showed that if $E$ is uniformly convex then $T$ has a fixed point, while Kirk [13] proved that if $E$ is reflexive and if $C$ has normal structure then $T$ has a fixed point. On the other hand, Goebel [7] defined the characteristic $\varepsilon_{0}$ of convexity of $E$ and showed that $E$ is uniformly convex if and only if $\varepsilon_{0}=0$, if $\varepsilon_{0}<1$ then $E$ has normal structure and if $\varepsilon_{0}<2$ then $E$ is reflexive. Also, Bynum [3] defined the normal structure coefficient $N(E)$ of $E$, and then Maluta [17] and Bae [1] proved that if $N(E)^{-1}<1$ then $E$ is reflexive and has normal structure. Using these coefficients, Goebel and Kirk [8], Goebel, Kirk and Thele [9] and Casini and Maluta [4] proved the fixed point theorems for uniformly $k$-lipschitzian mappings. (For the results on Hilbert space, see [5], [12], [14].) But it seems natural to define these coefficients for a convex set, since for any Banach space $E$, a nonexpansive mapping has a fixed point if $C$ is weakly compact and has normal structure.

In this paper, we introduce the modulus $\delta(C, \varepsilon)$ of convexity, the characteristic $\varepsilon_{0}(C)$ of convexity and the constant $\tilde{N}(C)$ of uniformity of normal structure for a convex subset $C$ of a Banach space and prove some results similar to [3], [7], [11], [17]. For example, we show that if $\tilde{N}(C)<1$ then $C$ is boundedly weakly compact. Further, by using these coefficients, we prove three fixed point theorems. All of these proofs are given by explicitly constructing a sequence which converges to a fixed point. We first show a fixed point theorem for nonexpansive semigroups. Secondly, we obtain a fixed point theorem for uniformly $k$-lipschitzian semigroups on $C$ under $k<\gamma$, where $\gamma$ is determined by the modulus of convexity of $C$. Also, using our results, we evaluate $\gamma$ as $1<\gamma \leqq 1+\left(1-\varepsilon_{0}(C)\right) / 2$. Finally, we prove that Casini and Maluta's result $[4]$ is valid under more general semigroups.

\section{§ 2. Preliminaries.}

Let $E$ be a real Banach space and let $B$ be a bounded subset of $E$. For a

Received November 12, 1986 
nonempty subset $C$ of $E$ define,

$$
\begin{aligned}
& R(B, x)=\sup \{\|x-y\|: y \in B\} \\
& R(B, C)=\inf \{R(B, x): x \in C\} \\
& \mathcal{C}(B, C)=\{x \in C: R(B, x)=R(B, C)\} .
\end{aligned}
$$

We call the number $R(B, C)$ the Chebyshev radius of $B$ in $C$ and the set $\mathcal{C}(C, B)$ the Chebyshev center of $B$ in $C$.

Let $\left\{B_{\alpha}: \alpha \in \Lambda\right\}$ be a decreasing net of bounded subsets of $E$. For a nonempty subset $C$ of $E$ define,

$$
\begin{gathered}
r\left(\left\{B_{\alpha}\right\}, x\right)=\inf _{\alpha} R\left(B_{\alpha}, x\right) ; \\
r\left(\left\{B_{\alpha}\right\}, C\right)=\inf \left\{r\left(\left\{B_{\alpha}\right\}, x\right): x \in C\right\} ; \\
\mathcal{A}\left(\left\{B_{\alpha}\right\}, C\right)=\left\{x \in C: r\left(\left\{B_{\alpha}\right\}, x\right)=r\left(\left\{B_{\alpha}\right\}, C\right)\right\} .
\end{gathered}
$$

The number $r\left(\left\{B_{\alpha}\right\}, C\right)$ and the set $\mathcal{A}\left(\left\{B_{\alpha}\right\}, C\right)$ are called the asymptotic radius and the asymptotic center of $\left\{B_{\alpha}: \alpha \in \Lambda\right\}$ in $C$, respectively. We also know that $R(B, \cdot)$ and $r\left(\left\{B_{\alpha}\right\}, \cdot\right)$ are continuous convex functions on $E$ which satisfy the following :

$$
\begin{aligned}
& |R(B, x)-R(B, y)| \leqq\|x-y\| \leqq R(B, x)+R(B, y) \\
& \left|r\left(\left\{B_{\alpha}\right\}, x\right)-r\left(\left\{B_{\alpha}\right\}, y\right)\right| \leqq\|x-y\| \leqq r\left(\left\{B_{\alpha}\right\}, x\right)+r\left(\left\{B_{\alpha}\right\}, y\right)
\end{aligned}
$$

for each $x, y \in E$, cf. [16].

A nonempty subset $C$ of $E$ is boundedly weakly compact if its intersection with every closed ball is weakly compact. It is easy to see that if $C$ is boundedly weakly compact and convex, then $\mathcal{C}(B, C)$ and $\mathcal{A}\left(\left\{B_{\alpha}\right\}, C\right)$ are nonempty.

For a subset $D$ of $E$, we denote by $d(D)$ the diameter of $D$ and by $\overline{c o} D$ the closure of the convex hull of $D$. A convex set $C$ of $E$ is said to have normal structure if each bounded convex subset $D$ of $C$ with $d(D)>0$ contains a point $y$ such that $R(D, y)<d(D)$.

The modulus of convexity of $E$ is the function

$$
\delta_{E}(\varepsilon)=\inf \left\{1-\left\|\frac{x+y}{2}\right\|:\|x\| \leqq 1,\|y\| \leqq 1,\|x-y\| \geqq \varepsilon\right\}
$$

defined for $0 \leqq \varepsilon \leqq 2$.

Let $S$ be a semitopological semigroup, i. e., $S$ is a semigroup with a Hausdorff topology such that for each $a \in S$ the mappings $s \rightarrow a \cdot s$ and $s \rightarrow s \cdot a$ from $S$ to $S$ are continuous. Let $C$ be a nonempty closed convex subset of $E$. Then a family $\mathcal{S}=\left\{T_{t}: t \in S\right\}$ of mappings from $C$ into itself is said to be aniformly $k$-lipschitzian semigroup on $C$ if $\mathcal{S}$ satisfies the following:

(1) $T_{t s}(x)=T_{t} T_{s}(x)$ for $t, s \in S$ and $x \in C$;

(2) the mapping $(s, x) \rightarrow T_{s}(x)$ from $S \times C$ into $C$ is continuous when $S \times C$ has 
the product topology;

(3) $\left\|T_{s}(x)-T_{s}(y)\right\| \leqq k\|x-y\|$ for $x, y \in C$ and $s \in S$.

In particular, a uniformly 1-lipschitzian semigroup on $C$ is said to be a nonexpansive semigroup on $C$. A semitopological semigroup $S$ is left reversible if any two closed right ideals of $S$ have nonvoid intersection. In this case, $(S, \leqq)$ is a directed system when the binary relation "@” on $S$ is defined by $a \leqq b$ if and only if $\{a\} \cup \overline{a S} \supseteqq\{b\} \cup \overline{b S}$.

\section{$\S 3$. Modulus of convexity and characteristic of convexity.}

We first define the modulus of convexity, the characteristic of convexity and the constant of uniformity of normal structure for a nonempty convex subset of a Banach space.

Definition 3.1. Let $C$ be a nonempty convex subset of a real Banach space $E$ with $d(C)>0$. Then we define, for $\varepsilon$ with $0 \leqq \varepsilon \leqq 2$,

$$
\begin{aligned}
& \delta(C, \varepsilon)=\inf \left\{1-\frac{1}{r}\left\|z-\frac{x+y}{2}\right\|: x, y, z \in C, 0<r \leqq d(C),\right. \\
& \qquad z-x\|\leqq r,\| z-y\|\leqq r,\| x-y \| \geqq r \varepsilon\} ; \\
& \varepsilon_{0}(C)=\sup \{\varepsilon: 0 \leqq \varepsilon \leqq 2, \delta(C, \varepsilon)=0\} ; \\
& \tilde{N}(C)=\sup \left\{\frac{R(D, D)}{d(D)}: D\right. \text { is a nonempty bounded convex } \\
& \text { subset of } C \text { with } d(D)>0\} .
\end{aligned}
$$

Remark 3.1. It follows from Definition 3.1 that $\delta(C, 0)=0,0 \leqq \delta(C, \varepsilon) \leqq 1$, $\delta(C, \varepsilon)$ is nondecreasing in $\varepsilon$ and $\delta(E, \varepsilon)=\delta_{E}(\varepsilon)$. Further for a nonempty convex subset $D$ of $C$ with $d(D)>0$ it follows that $\delta(C, \varepsilon) \leqq \delta(D, \varepsilon), \varepsilon_{0}(D) \leqq \varepsilon_{0}(C)$ and $\tilde{N}(D) \leqq \tilde{N}(C)$.

Remark 3.2. Let $C$ and $D$ be convex subsets of $E$. For $a \in E$, it is easy to see that $\delta(\bar{C}, \varepsilon)=\delta(C, \varepsilon), \delta(C+a, \varepsilon)=\delta(C, \varepsilon)$, and $\delta(C \cap D, \varepsilon)=\max \{\delta(C, \varepsilon)$, $\delta(D, \varepsilon)\}$. Similarly we have $\tilde{N}(\bar{C})=\tilde{N}(C), \tilde{N}(C+a)=\tilde{N}(C)$, and $\tilde{N}(C \cap D)=$ $\min \{\tilde{N}(C), \tilde{N}(D)\}$.

Example 3.1. Let $C[0,1]$ be a Banach space of all continuous real functions on $[0,1]$ with supremum norm and let $A$ be a subspace of all affine functions in $C[0,1]$. Since $C[0,1]$ is not reflexive, we have $\tilde{N}(C[0,1])=1$. But it is easy to see that $A$ is isomorphic to $l_{\infty}^{2}=\left(\boldsymbol{R}^{2},\|\cdot\|_{\infty}\right)$ and hence $\tilde{N}(A)=\tilde{N}\left(l_{\infty}^{2}\right)=$ $\frac{1}{2}$, cf. [17], [1]. 
It is well known that $\delta_{E}(\varepsilon)$ is continuous on $[0,2)$, cf $[11]$. We can also prove an inequality concerning the continuity of $\delta(C, \varepsilon)$. Before proving it we need the following lemma.

LEMMA 3.1. Let $C$ be a nonempty convex subset of a real Banach space $E$ with $d(C)>0$, let $u, v \in E$ and let $0<r \leqq d(C)$. For $z \in C$ and $\varepsilon$ with $0 \leqq \varepsilon \leqq 2$ define $a$ set $N_{r, u, v}(z)$ and a function $\delta_{r, u, v}(\varepsilon)$ as follows:

$$
\begin{aligned}
& N_{r, u, v}(z)=\{(x, y): x, y \in C,\|z-x\| \leqq r,\|z-y\| \leqq r, \\
& \left.x-y=a u, z-\frac{x+y}{2}=b v \text { for some } a, b \geqq 0\right\} ; \\
& \delta_{r, u, v}(\varepsilon)=\inf \left\{1-\frac{1}{r}\left\|z-\frac{x+y}{2}\right\|: z \in C,(x, y) \in N_{r, u, v}(z),\|x-y\| \geqq r \varepsilon\right\} .
\end{aligned}
$$

Then $\boldsymbol{\delta}_{r, u, v}$ is a nondecreasing convex function from $[0,2]$ to $[0,1]$ with

$$
\delta(C, \varepsilon)=\inf \left\{\delta_{r, u, v}(\varepsilon): u, v \in E, 0<r \leqq d(C)\right\} .
$$

Proof. Since it is obvious that $\delta_{r, u, v}$ is nondecreasing and

$$
\delta(C, \varepsilon)=\inf \left\{\delta_{r, u, v}(\varepsilon): u, v \in E, 0<r \leqq d(C)\right\},
$$

we only prove that $\delta_{r, u, v}$ is convex.

For arbitrary $z_{1}, z_{2} \in C$ and $\left(x_{1}, y_{1}\right) \in N_{r, u, v}\left(z_{1}\right)$ and $\left(x_{2}, y_{2}\right) \in N_{r, u, v}\left(z_{2}\right)$ with $\left\|x_{1}-y_{1}\right\| \geqq r \varepsilon_{1}$ and $\left\|x_{2}-y_{2}\right\| \geqq r \varepsilon_{2}$, there exist $a_{1}, a_{2}, b_{1}, b_{2} \geqq 0$ such that

$$
x_{1}-y_{1}=a_{1} u, z_{1}-\frac{x_{1}+y_{1}}{2}=b_{1} v \text {, }
$$

and

$$
x_{2}-y_{2}=a_{2} u, z_{2}-\frac{x_{2}+y_{2}}{2}=b_{2} v \text {. }
$$

For $\lambda$ with $0 \leqq \lambda \leqq 1$, define $x_{3}=\lambda x_{1}+(1-\lambda) x_{2}, y_{3}=\lambda y_{1}+(1-\lambda) y_{2}$ and $z_{3}=\lambda z_{1}+$ $(1-\lambda) z_{2}$. Then, we have

$$
\begin{gathered}
x_{3}-y_{3}=\lambda\left(x_{1}-y_{1}\right)+(1-\lambda)\left(x_{2}-y_{2}\right)=\left(\lambda a_{1}+(1-\lambda) a_{2}\right) u, \\
z_{3}-\frac{x_{3}+y_{3}}{2}=\lambda\left(z_{1}-\frac{x_{1}+y_{1}}{2}\right)+(1-\lambda)\left(z_{2}-\frac{x_{2}+y_{2}}{2}\right) \\
=\left(\lambda b_{1}+(1-\lambda) b_{2}\right) v .
\end{gathered}
$$

Since $\left\|z_{3}-x_{3}\right\| \leqq r$ and $\left\|z_{3}-y_{3}\right\| \leqq r$, we have $\left(x_{3}, y_{3}\right) \in N_{r, u, v}\left(z_{3}\right)$. We also obtain

$$
\left\|x_{3}-y_{3}\right\|=\lambda\left\|x_{1}-y_{1}\right\|+(1-\lambda)\left\|x_{2}-y_{2}\right\| \geqq \lambda \varepsilon_{1}+(1-\lambda) \varepsilon_{2}
$$

and 


$$
\begin{aligned}
\delta_{r, u, v}\left(\lambda \varepsilon_{1}+(1-\lambda) \varepsilon_{2}\right) \leqq 1-\frac{1}{r}\left\|z_{3}-\frac{x_{3}+y_{3}}{2}\right\| \\
\quad=\lambda\left(1-\frac{1}{r}\left\|z_{1}-\frac{x_{1}+y_{1}}{2}\right\|\right)+(1-\lambda)\left(1-\frac{1}{r}\left\|z_{2}-\frac{x_{2}+y_{2}}{2}\right\|\right)
\end{aligned}
$$

for arbitrary $z_{1}, z_{2} \in C,\left(x_{1}, y_{1}\right) \in N_{r, u, v}\left(z_{1}\right)$ and $\left(x_{2}, y_{2}\right) \in N_{r, u, v}\left(z_{2}\right)$. Therefore we have

$$
\delta_{r, u, v}\left(\lambda \varepsilon_{1}+(1-\lambda) \varepsilon_{2}\right) \leqq \lambda \delta_{r, u, v}\left(\varepsilon_{1}\right)+(1-\lambda) \delta_{r, u, v}\left(\varepsilon_{2}\right) .
$$

THEOREM 3.1. Let $C$ be a nonempty convex subset of a real Banach space $E$ with $d(C)>0$. Then for all $\varepsilon_{1}$ and $\varepsilon_{2}$ with $0 \leqq \varepsilon_{1}<\varepsilon_{2} \leqq 2$,

$$
\delta\left(C, \varepsilon_{2}\right)-\delta\left(C, \varepsilon_{1}\right) \leqq \frac{\varepsilon_{2}-\varepsilon_{1}}{2-\varepsilon_{1}}\left(1-\delta\left(C, \varepsilon_{1}\right)\right) \leqq \frac{\varepsilon_{2}-\varepsilon_{1}}{2-\varepsilon_{1}} .
$$

Proof. For any real number with $\eta>0$, there exist $u, v \in E$ and $r$ with $0<r<d(C)$ such that $\delta_{r, u, v}\left(\varepsilon_{1}\right) \leqq \delta\left(C, \varepsilon_{1}\right)+\eta$ and hence we obtain

$$
\begin{aligned}
\delta_{r, u, v}\left(\varepsilon_{2}\right) & =\delta_{r, u, v}\left(\left(\frac{\varepsilon_{2}-\varepsilon_{1}}{2-\varepsilon_{1}}\right) 2+\left(1-\frac{\varepsilon_{2}-\varepsilon_{1}}{2-\varepsilon_{1}}\right) \varepsilon_{1}\right) \\
& \leqq \frac{\varepsilon_{2}-\varepsilon_{1}}{2-\varepsilon_{1}} \delta_{r, u, v}(2)+\left(1-\frac{\varepsilon_{2}-\varepsilon_{1}}{2-\varepsilon_{1}}\right) \delta_{r, u, v}\left(\varepsilon_{1}\right)
\end{aligned}
$$

or

$$
\begin{aligned}
\delta_{r, u, v}\left(\varepsilon_{2}\right)-\delta_{r, u, v}\left(\varepsilon_{1}\right) & \leqq \frac{\varepsilon_{2}-\varepsilon_{1}}{2-\varepsilon_{1}}\left(\delta_{r, u, v}(2)-\delta_{r, u, v}\left(\varepsilon_{1}\right)\right) \\
& \leqq \frac{\varepsilon_{2}-\varepsilon_{1}}{2-\varepsilon_{1}}\left(1-\delta\left(C, \varepsilon_{1}\right)\right) .
\end{aligned}
$$

Then we have

$$
\begin{aligned}
\delta\left(C, \varepsilon_{2}\right)-\delta\left(C, \varepsilon_{1}\right) & \leqq \delta_{r, u, v}\left(\varepsilon_{2}\right)-\delta_{r, u, v}\left(\varepsilon_{1}\right)+\eta \\
& \leqq \frac{\varepsilon_{2}-\varepsilon_{1}}{2-\varepsilon_{1}}\left(1-\delta\left(C, \varepsilon_{1}\right)\right)+\eta .
\end{aligned}
$$

Since $\eta>0$ is arbitrary, we have

$$
\delta\left(C, \varepsilon_{2}\right)-\delta\left(C, \varepsilon_{1}\right) \leqq \frac{\varepsilon_{2}-\varepsilon_{1}}{2-\varepsilon_{1}}\left(1-\delta\left(C, \varepsilon_{1}\right)\right) \leqq \frac{\varepsilon_{2}-\varepsilon_{1}}{2-\varepsilon_{1}} .
$$

The following lemma can be proved as in [16].

LEMMA 3.2. Let $C$ be a convex subset of a real Banach space $E$. Let $B$ be $a$ bounded subset of $C$ and let $\left\{B_{\alpha}: \alpha \in \Lambda\right\}$ be a decreasing net of bounded subsets of $C$. For each $x, y \in C$, if $R(B, x) \leqq t, R(B, y) \leqq t$ and $\|x-y\| \geqq t \cdot \varepsilon$ then 


$$
R\left(B, \frac{x+y}{2}\right) \leqq t(1-\delta(C, \varepsilon))
$$

and if $r\left(\left\{B_{\alpha}\right\}, x\right) \leqq t, r\left(\left\{B_{\alpha}\right\}, y\right) \leqq t$ and $\|x-y\| \geqq t \varepsilon$ then

$$
r\left(\left\{B_{\alpha}\right\}, \frac{x+y}{2}\right) \leqq t(1-\delta(C, \varepsilon)) .
$$

It was proved by Bynum [3] that $\tilde{N}(E) \leqq 1-\delta_{E}(1)$. By using Theorem 3.1, Lemma 3.2 and the method of [3], we can also obtain the following: Let $C$ be a nonempty convex subset of a real Banach space $E$ with $d(C)>0$. Then $\tilde{N}(C)$ $\leqq 1-\delta(C, 1)$.

Maluta [17] and Bae [1] proved that if $\tilde{N}(E)<1$ then $E$ is reflexive. We can prove the following:

THEOREM 3.2. Let $C$ be a nonempty convex subset of a real Banach space $E$ with $d(C)>0$. If $\tilde{N}(C)<1$ then $C$ is boundedly weakly compact and has normal structure.

Proof. It is obvious from $\tilde{N}(C)<1$ that $C$ has normal structure. We may assume that $C$ is bounded. Let $\left\{C_{n}\right\}$ be an arbitrary decreasing sequence of nonempty closed convex subsets of $C$. If we show $\left\{C_{n}\right\}$ has nonempty intersection then we complete the proof, cf. [p. 433,4]. If $d\left(C_{n}\right)=0$ for some $n \geqq 1$ then it is obvious that $\left\{C_{n}\right\}$ has nonempty intersection. So we assume $d\left(C_{n}\right)>0$ for all $n \geqq 1$. Let $\eta$ be a real number with $\tilde{N}(C)<\eta<1$ and define by induction:

$$
\begin{aligned}
& C_{n, 0}=C_{n} ; \\
& x_{n, m} \in C_{n, m} \text { such that } R\left(C_{n, m}, x_{n, m}\right) \leqq \eta d\left(C_{n, m}\right) ; \\
& C_{n, m+1}=\overline{c o}\left\{x_{k, m}: k \geqq n\right\} .
\end{aligned}
$$

Then, we have $C_{n, m}$ is nonempty, $C_{n, m} \supseteqq C_{n+1, m}, C_{n, m} \supseteqq C_{n, m+1}$ and

$$
\begin{aligned}
d\left(C_{n, m}\right) & =\sup \left\{\left\|x_{\imath, m-1}-x_{j, m-1}\right\|: i, j \geqq n\right\}=\sup _{i \geqq n} \sup _{j \geqq i}\left\|x_{\imath, m-1}-x_{\jmath, m-1}\right\| \\
& \leqq \sup _{i \geq n} R\left(C_{i, m}, x_{\imath, m-1}\right) \leqq \sup _{i \geq n} R\left(C_{i, m-1}, x_{\imath, m-1}\right) \\
& \leqq \sup _{i \geqq n} \eta d\left(C_{\imath, m-1}\right) \leqq \eta d\left(C_{n, m-1}\right) \leqq \eta^{m} d\left(C_{n}\right)
\end{aligned}
$$

for all $n, m \geqq 1$. Hence $\lim _{m \rightarrow \infty} d\left(C_{n, m}\right)=0$. Since $\bigcap_{m=1}^{\infty} C_{n, m} \supseteqq \bigcap_{m=1}^{\infty} C_{n+1, m}$ for all $n \geqq 1$, there exists $y \in E$ such that $\bigcap_{m=1}^{\infty} C_{n, m}=\{y\}$ for all $n \geqq 1$. Therefore $\bigcap_{n=1}^{\infty} C_{n}$ is nonempty.

Corollary 3.1 (Maluta [17] and Bae [1]). Let $E$ be a real Banach space with $\tilde{N}(E)<1$. Then $E$ is reflexive and has normal structure. 


\section{$\S 4$. Fixed point theorems.}

In this section, we prove three fixed point theorems by using the results obtained in section 3. The following lemma is crucial in the proofs.

LEMMA 4.1. Let $C$ be a convex subset of a real Banach space E. Let $\left\{B_{\alpha}\right.$ : $\alpha \in \Lambda\}$ be a decreasing net of bounded subsets of $C$ and let $D$ be $a$ boundedly weakly compact convex subset of $C$. Let $r$ be the asymptotic radius and $A$ be the asymptotic center of $\left\{B_{\alpha}\right\}$ in $D$. Then

$$
d(A) \leqq \varepsilon_{0}(C) r .
$$

Further let $\varepsilon_{0}(C)<1$ and let $\gamma$ be a real number such that $\gamma(1-\delta(C, 1 / \gamma))=1$. For a real number $k$ with $1 \leqq k<\gamma$, define $A_{k}=\left\{x \in D: r\left(\left\{B_{\alpha}\right\}, x\right) \leqq k r\right\}$. Then

$$
d\left(A_{k}\right) \leqq \frac{k}{\gamma} r .
$$

Proof. In case $r=0$, the inequality is true. In fact, if $x, y \in A$ then

$$
\|x-y\| \leqq r\left(\left\{B_{\alpha}\right\}, x\right)+r\left(\left\{B_{\alpha}\right\}, y\right)=0
$$

and hence $d(A)=0$. So we assume $r>0$ and $d(A)>0$. For any real number $\eta$ with $0<\eta<d(A)$, there exist $x, y \in A$ such that $\|x-y\| \geqq d(A)-\eta$. By Lemma 3.2 and convexity of $A$, we have

$$
r=r\left(\left\{B_{\alpha}\right\}, \frac{x+y}{2}\right) \leqq r\left(1-\delta\left(C, \frac{d(A)-\eta}{r}\right)\right) .
$$

This implies

$$
\delta\left(C, \frac{d(A)-\eta}{r}\right)=0
$$

and hence $d(A) \leqq \varepsilon_{0}(C) r$.

We may also assume $r>0$ and $d\left(A_{k}\right)>0$. For any real number $\eta$ with $0<$ $\eta<d\left(A_{k}\right)$, there exist $x, y \in A_{k}$ such that $\|x-y\| \geqq d\left(A_{k}\right)-\eta$. Then, we have

$$
r \leqq r\left(\left\{B_{\alpha}\right\}, \frac{x+y}{2}\right) \leqq k r\left(1-\delta\left(C, \frac{d\left(A_{k}\right)-\eta}{k r}\right)\right) .
$$

Since $\eta>0$ is arbitrary and $\delta$ is continuous, it follows that

$$
\delta\left(C, \frac{d\left(A_{k}\right)}{k r}\right) \leqq 1-\frac{1}{k} \text {. }
$$

Suppose that $\frac{1}{r} \leqq \frac{d\left(A_{k}\right)}{k r}$. Then we have

$$
1-\frac{1}{\gamma}=\delta\left(C, \frac{1}{\gamma}\right) \leqq \delta\left(C, \frac{d\left(A_{k}\right)}{k r}\right) \leqq 1-\frac{1}{k}<1-\frac{1}{\gamma} .
$$


This is a contradiction.

Remark 4.1. From Lemma 4.1, we have immediately the similar inequality concerning the Chebyshev radius and center. In fact, putting $B_{\alpha}=B$, we have

$$
d(\mathcal{C}(B, D)) \leqq \varepsilon_{0}(C) R(B, C) .
$$

The following theorem is a special case of results of Lim [15] and Takahashi [18], while the proof is constructive.

THEOREM 4.1. Let $C$ be a closed convex subset of a real Banach space $E$ with $\varepsilon_{0}(C)<1$ and let $\mathcal{S}=\left\{T_{t}: t \in S\right\}$ be a nonexpansive semigroup on $C$. Suppose that $S$ is left reversible and $\left\{T_{t} y: t \in S\right\}$ is bounded for some $y \in C$. Then there exists a $z \in C$ such that $T_{s} z=z$ for all $s \in S$.

Proof. Let $B_{s}(x)=\left\{T_{t} x: t \geqq s\right\}$ for $s \in S$ and $x \in C$. Define $\left\{x_{n}: n \geqq 0\right\}$ by induction as follows:

$$
\begin{aligned}
& x_{0}=y ; \\
& x_{n} \in \mathcal{A}\left(\left\{B_{s}\left(x_{n-1}\right)\right\}, C\right) \quad \text { for } n \geqq 1 .
\end{aligned}
$$

Let $r_{n}(x)=r\left(\left\{B_{s}\left(x_{n-1}\right)\right\}, x\right), r_{n}=r\left(\left\{B_{s}\left(x_{n-1}\right)\right\}, C\right)$ and $A_{n}=\mathcal{A}\left(\left\{B_{s}\left(x_{n-1}\right)\right\}, C\right)$ for $n \geqq 1$. Then we have

$$
\begin{aligned}
r_{n}\left(T_{t} x_{n}\right) & =\lim _{s} \sup \left\|T_{s} x_{n-1}-T_{t} x_{n}\right\| \leqq \limsup _{s}\left\|T_{t} T_{s} x_{n-1}-T_{t} x_{n}\right\| \\
& \leqq \lim _{s} \sup \left\|T_{s} x_{n-1}-x_{n}\right\|=r_{n}
\end{aligned}
$$

for all $t \in S$ and $n \geqq 1$ and hence $T_{t} A_{n} \subseteq A_{n}$ for $t \in S$ and $n \geqq 1$. By Lemma 4.1, we obtain

$$
\begin{aligned}
r_{n+1} & =r_{n+1}\left(x_{n+1}\right) \leqq r_{n+1}\left(x_{n}\right) \leqq \sup _{s}\left\|T_{s} x_{n}-x_{n}\right\| \\
& \leqq d\left(A_{n}\right) \leqq \varepsilon_{0}(C) r_{n} \leqq\left(\varepsilon_{0}(C)\right)^{n} r_{1}
\end{aligned}
$$

and hence

$$
\begin{aligned}
\left\|x_{n+1}-x_{n}\right\| & \leqq r\left(\left\{B_{s}\left(x_{n}\right)\right\}, x_{n+1}\right)+r\left(\left\{B_{s}\left(x_{n}\right)\right\}, x_{n}\right)=r_{n+1}+r_{n+1}\left(x_{n}\right) \\
& \leqq 2\left(\varepsilon_{0}(C)\right)^{n} r_{1}
\end{aligned}
$$

for all $n \geqq 1$. So, $\left\{x_{n}\right\}$ is a Cauchy sequence and hence $\left\{x_{n}\right\}$ converges to a point $z \in C$. Therefore we have

$$
\begin{aligned}
\left\|z-T_{s} z\right\| & =\lim _{n \rightarrow \infty}\left\|x_{n}-T_{s} x_{n}\right\| \leqq \lim _{n \rightarrow \infty}\left(r_{n}\left(x_{n}\right)+r_{n}\left(T_{s} x_{n}\right)\right) \\
& \leqq \lim _{n \rightarrow \infty} 2\left(\varepsilon_{0}(C)\right)^{n-1} r_{1}=0
\end{aligned}
$$

for all $s \in S$.

By the method of Theorem 4.1, we can prove the following fixed point theorem which is slightly different from [9]. 
THEOREM 4.2. Let $C$ be a closed convex subset of a real Banach space $E$ with $\varepsilon_{0}(C)<1$ and let $\gamma$ be a real number such that $\gamma(1-\delta(C, 1 / \gamma))=1$. Let $\mathcal{S}=$ $\left\{T_{t}: t \in S\right\}$ be a uniformly $k$-lipschitzian semigroup on $C$ with $1 \leqq k<\gamma$. Suppose that $S$ is left reversible and $\left\{T_{t} y: t \in S\right\}$ is bounded for some $y \in C$. Then there exists a $z \in C$ such that $T_{s} z=z$ for all $s \in S$.

Proof. Let $B_{s}(x)=\left\{T_{t} x: t \geqq s\right\}$ for $s \in S$ and $x \in C$. Define $\left\{x_{n}: n \geqq 0\right\}$ by induction as follows:

$$
\begin{aligned}
& x_{0}=y ; \\
& x_{n} \in \mathcal{A}\left(\left\{B_{s}\left(x_{n-1}\right)\right\}, C\right) \text { for } n \geqq 1 .
\end{aligned}
$$

Let $r_{n}(x)=r\left(\left\{B_{s}\left(x_{n-1}\right)\right\}, x\right), r_{n}=r\left(\left\{B_{s}\left(x_{n-1}\right)\right\}, C\right)$ and $A_{n}=\left\{x \in C: r_{n}(x) \leqq k r_{n}\right\}$ for $n \geqq 1$. Then since $r_{n}\left(x_{n}\right)=r_{n} \leqq k r_{n}$ and

$$
r_{n}\left(T_{t} x_{n}\right)=\limsup _{s}\left\|T_{s} x_{n-1}-T_{t} x_{n}\right\| \leqq k \lim _{s} \sup \left\|T_{s} x_{n-1}-x_{n}\right\|=k r_{n}
$$

for all $t \in S$ and $n \geqq 1$, we have $x_{n}, T_{t} x_{n} \in A_{n}$ for all $t \in S$ and $n \geqq 1$. By Lemma 4.1 , we obtain

$$
\begin{gathered}
r_{n+1}=r_{n+1}\left(x_{n+1}\right) \leqq r_{n+1}\left(x_{n}\right) \leqq \sup _{s}\left\|T_{s} x_{n}-x_{n}\right\| \\
\leqq d\left(A_{n}\right) \leqq \frac{k}{\gamma} r_{n} \leqq\left(\frac{k}{\gamma}\right)^{n} r_{1}
\end{gathered}
$$

for all $n \geqq 1$. Therefore, as in the proof of Theorem 4.1, $\left\{x_{n}\right\}$ converges to a point $z \in C$. So, we have

$$
\begin{gathered}
\left\|z-T_{s} z\right\|=\lim _{n \rightarrow \infty}\left\|x_{n}-T_{s} x_{n}\right\| \leqq \lim _{n \rightarrow \infty}\left(r_{n}\left(x_{n}\right)+r_{n}\left(T_{s} x_{n}\right)\right) \\
\leqq \lim _{n \rightarrow \infty}(1+k) r_{n}=0
\end{gathered}
$$

for all $s \in S$.

Remark 4.2. Let $C$ and $\gamma$ be defined as in Theorem 4.2. Then we have

$$
1<\gamma \leqq 1+\frac{1-\varepsilon_{0}(C)}{2} .
$$

In fact, let $\eta=1 / \gamma$ and if $\delta(C, \eta)=1-\eta=0$. Then we have $1>\varepsilon_{0}(C) \geqq \eta=1$. This is a contradiction. Hence $\varepsilon_{0}(C) \leqq \eta<1$. So, from Theorem 3.1,

$$
1-\eta=\delta(C, \eta) \leqq \frac{\eta-\varepsilon_{0}(C)}{2-\varepsilon_{0}(C)} .
$$

Therefore we have

$$
1<\gamma \leqq 1+\frac{1-\varepsilon_{0}(C)}{2} .
$$


We can also obtain a generalization of Casini and Maluta's fixed point theorem [4].

LEMMA 4.2. Let $C$ be a boundedly weakly compact convex subset of a real Banach space $E$. Let $\left\{B_{\alpha}: \alpha \in \Lambda\right\}$ be a decreasing net of nonempty bounded closed convex subsets of $C$ and let $B=\bigcap_{\alpha} B_{\alpha}$. Then

$$
r\left(\left\{B_{\alpha}\right\}, B\right) \leqq \tilde{N}(C) \inf _{\alpha} d\left(B_{\alpha}\right) .
$$

Proof. Let $u_{\beta} \in \mathcal{C}\left(B_{\beta}, B_{\beta}\right)$ for each $\beta \in \Lambda$. Then we have

$$
r\left(\left\{B_{\alpha}\right\}, u_{\beta}\right) \leqq R\left(B_{\beta}, u_{\beta}\right)=R\left(B_{\beta}, B_{\beta}\right) \leqq \tilde{N}(C) d\left(B_{\beta}\right) .
$$

Let $\left\{u_{\beta_{\gamma}}\right\}$ be a subnet of $\left\{u_{\beta}\right\}$ which converges weakly to a point $u_{0} \in B$. By weakly lower semicontinuity of $r$ and monotonicity of $d\left(B_{\beta}\right)$, we have

$$
\begin{aligned}
r\left(\left\{B_{\alpha}\right\}, B\right) & \leqq r\left(\left\{B_{\alpha}\right\}, u_{0}\right) \leqq \liminf _{\gamma} r\left(\left\{B_{\alpha}\right\}, u_{\beta_{\gamma}}\right) \leqq \liminf _{\gamma} \tilde{N}(C) d\left(B_{\beta_{\gamma}}\right) \\
& =\tilde{N}(C) \inf _{\gamma} d\left(B_{\beta_{\gamma}}\right)=\tilde{N}(C) \inf _{\alpha} d\left(B_{\alpha}\right) .
\end{aligned}
$$

THEOREM 4.3. Let $C$ be a closed convex subset of a real Banach space $E$ with $\tilde{N}(C)<1$ and let $\mathcal{S}=\left\{T_{t}: t \in S\right\}$ be a uniformly k-lipschitzian semigroup on $C$ with $k<\tilde{N}(C)^{-1 / 2}$. Suppose that $S$ is left reversible and $\left\{T_{t} y: t \in S\right\}$ is bounded for some $y \in C$. Then there exists a $z \in C$ such that $T_{s} z=z$ for all $s \in S$.

Proof. Let $B_{s}(x)=\overline{c o}\left\{T_{t} x: t \geqq s\right\}$ and let $B(x)=\bigcap_{s} B_{s}(x)$ for $s \in S$ and $x \in C$. Define $\left\{x_{n}: n \geqq 0\right\}$ by induction as follows :

$$
\begin{aligned}
& x_{0}=y ; \\
& x_{n} \in \mathcal{A}\left(\left\{B_{s}\left(x_{n-1}\right)\right\}, B\left(x_{n-1}\right)\right) \quad \text { for } n \geqq 1 .
\end{aligned}
$$

Let $r_{n}(x)=r\left(\left\{B_{s}\left(x_{n-1}\right)\right\}, x\right)$ and $r_{n}=r\left(\left\{B_{s}\left(x_{n-1}\right)\right\}, B\left(x_{n-1}\right)\right)$ for $n \geqq 1$. Then from $x_{n} \in B\left(x_{n-1}\right)=\bigcap_{t} B_{t}\left(x_{n-1}\right)$ for $n \geqq 1$, we have

$$
\begin{aligned}
r_{n+1}\left(x_{n}\right) & =\lim _{s} \sup \left\|T_{s} x_{n}-x_{n}\right\| \leqq \lim \sup _{s}\left(\inf _{t} R\left(B_{t}\left(x_{n-1}\right), T_{s} x_{n}\right)\right) \\
& =\lim \sup _{s} r_{n}\left(T_{s} x_{n}\right)=\lim \sup _{s}\left(\lim _{t} \sup \left\|T_{t} x_{n-1}-T_{s} x_{n}\right\|\right) \\
& \leqq \lim _{s} \sup \left(k \lim _{t} \sup \left\|T_{t} x_{n-1}-x_{n}\right\|\right)=k r_{n} \\
& \leqq k \tilde{N}(C) \inf _{s} d\left(B_{s}\left(x_{n-1}\right)\right)
\end{aligned}
$$

and

$$
\inf _{s} d\left(B_{s}\left(x_{n-1}\right)\right)=\inf _{s} \sup \left\{\left\|T_{a} x_{n-1}-T_{b} x_{n-1}\right\|: a, b \geqq s\right\}
$$


Hence we have

$$
\begin{aligned}
& \leqq \lim _{t} \sup \left(\lim _{s} \sup \left\|T_{s} x_{n-1}-T_{t} x_{n-1}\right\|\right) \\
& =\lim _{t} \sup r_{n}\left(T_{t} x_{n-1}\right) \\
& \leqq k r_{n}\left(x_{n-1}\right)
\end{aligned}
$$

$$
r_{n+1}\left(x_{n}\right) \leqq k r_{n} \leqq k^{2} \tilde{N}(C) r_{n}\left(x_{n-1}\right) \leqq\left(k^{2} \tilde{N}(C)\right)^{n} r_{1}\left(x_{0}\right) .
$$

Therefore, as in the proof of Theorem 4.2, $\left\{x_{n}\right\}$ converges to a common fixed point.

\section{REFERENCES}

[1] BAE, J.S., Reflexivity of a Banach space with a uniformly normal structure, Proc. Amer. Math. Soc. 90 (1984), 269-270.

「2] BRowder, F.E., Nonexpansive nonlinear operators in Banach space, Proc. Nat. Acad. Sci. U. S. A. 54 (1965), 1041-1044.

[3] Bynum, W.L., Normal structure coefficients for Banach spaces, Pacific J. Math. 86 (1980), 427-436.

[4] Casini, E. and E. Maluta, Fixed points of uniformly lipschitzian mappings in spaces with uniformly normal structure, Nonlinear Analysis 9 (1985), 103-108.

[5] Downing, D.J. AND W.O. RAY, Uniformly lipschitzian semigroup in Hilbert space, Canad. Math. Bull. 25 (1982), 210-214.

[6] Dunford, N. ANd J.T. Schwartz, Linear operators, Part 1., Interscience, New York (1958).

[7] Goebel, K., Convexity of balls and fixed-point theorems for mappings with nonexpansive square, Compositio Math. 22 Fasc. 3 (1970), 269-274.

[8] Goebel, K. AND W.A. KIRK, A fixed point theorem for transformations whose iterates have uniform Lipschitz constant, Studia Math. 47 (1973), 135-140.

[9] Goebel, K., W.A. Kirk and R.L. Thele, Uniformly lipschitzian families of transformations in Banach space, Can. J. Math. 26 (1974), 1245-1256.

[10] GöHDE, D., Zum prinzip der kontraktiven abbildung, Math. Nachr. 30 (1965), 251-258.

[11] GURARII, V.I., On the differential properties of the modulus of convexity in a Banach space, Mat. Issled. 2 (1967), 141-148.

[12] IshinARA, H. AND W. TAKAHASHI, Fixed point theorems for uniformly lipschitzian semigroups in Hilbert spaces, to appear in J. Math. Anal. Appl. 126 (1987).

[13] KIRK, W.A., A fixed point theorem for mappings which do not increase distance, Amer. Math. Monthly 72 (1965), 1004-1006.

[14] Lifschitz, E. A., Fixed point theorems for operators in strongly convex spaces, Voronez Gos. Univ. Trudy Math. Fak. 16 (1975), 23-28.

[15] Lim, T.C., Characterizations of normal structure, Proc. Amer. Math. Soc. 43 (1974), 313-319.

[16] LIM, T.C., On asymptotic centers and fixed points of nonexpansive mappings, Can. J. Math. 32 (1980), 421-430.

[17] Maluta, E., Uniformly normal structure and related coefficients, Pacific J. Math. 111 (1984), 357-369. 
[18] Takahashi, W., Fixed point theorems for families of nonexpansive mappings on unbounded sets, J. Math. Soc. Japan 36 (1984), 545-553.

Department of Information Science,

TOKyo Institute of TeCHNOLOGY

Oh-OKayama, Meguro-Ku, Tokyo 152, Japan 